\title{
Rapid identification of mycobacterial species directly in clinical samples
}

\author{
Raffaella Rossi', Fabio Mengoni', Ilaria Sauzullo', Miriam Lichtner', Laura Scorzolini', \\ Fernanda Chiarini', Vincenzo Vullo' \\ 'Dipartimento di Malattie Infettive e Tropicali, "Sapienza" Università di Roma. \\ 2Dipartimento di Scienze di Sanità Pubblica, "Sapienza” Università di Roma.
}

Key words: Non tuberculous mycobacteria, GenoType CM/AS, Reverse hybridization, PCR

\section{Diagnosi rapida d'infezione da Micobatteri non tubercolari su campioni respiratori}

\section{SUMMARY}

Up to today, the prompt diagnosis of NTM infection has been impaired by the slow growth in culture media, which is an essential step for proper identification.

The aim of our study was to evaluate the kit Genotype Mycobacterium CM/AS for the identification of NTM in clinical specimens. Four patients admitted to the Department of Infectious and Tropical Diseases of Sapienza University in Rome were included in the study. Three out of 4 patients were strongly suspected to have a NTM infection and one of them underwent treatment for M. tuberculosis infection without any substantial clinical improvement.

After decontamination of the specimens, the extracted DNA was amplified in target sequences using PCR assay by increasing the number of cycles from 20 to 30 . Subsequently the Genotype assay was performed according to the manufacturer's instructions. Our results confirmed the presence of NTM in all patients: M. peregrinum in two patients, $M$. gordone and $M$. intracellulare in the others.

The use of the Genotype Mycobacterium CM/AS directly from clinical specimens permits rapid diagnosis and enables clinicians to start an effective treatment.

\section{Received March 5, 2008}

Accepted May 26, 2008

\section{INTRODUZIONE}

Il genere Mycobacterium comprende più di 120 differenti specie di cui oltre un terzo sono considerate patogene per l'uomo $(3,11)$. I micobatteri patogeni vengono generalmente classificati in tre gruppi: Mycobacterium tuberculosis complex, cui appartengono le specie $M$. tuberculosis, $M$. africanum, $M$. bovis, M. microti, $M$. canetti; i micobatteri non tubercolari (MNT) ed infine un terzo gruppo cui appartiene il $M$. leprae. I micobatteri vengono, inoltre, distinti in base al tempo di crescita in coltura: micobatteri a crescita rapida $(\leq 7$ giorni) e micobatteri a lenta crescita (>7giorni). Il gruppo dei MNT comprende le specie che comunemente si ritrovano nell'ambiente o che utilizzano gli animali domestici come ospiti intermedi: sono tutte specie che raramente arrecano danno nell'individuo immunocompetente. Le categorie generalmente più a rischio d'infezione da micobatteri atipici sono i bambini al di sotto dei 5 anni, anche se immunocompetenti, e i pazienti immunocompromessi.

È noto, infatti, che prima dell'avvento dell'infezione da HIV i casi d'infezione da micobatteri atipici erano relativamente rari. Negli ultimi anni, infatti, si è assistito ad un aumento di tali infezioni in questi pazienti a causa, proprio, del loro stato di compromissione immunitaria. Oggi nei pazienti HIV positivi si osservano dal 25 al $50 \%$ di casi d'infezioni da micobatteri non tubercolari $(6,7)$. L'aumento delle infezioni da MNT è, inoltre, da attribuire anche all'aumento di pazienti con stato immunitario compromesso a causa di patologie immunitarie o neoplasie che richiedono terapie immunosoppressive.

I maggiori problemi di studio e di tipizzazione dei micobatteri è legato alle specie che hanno lunghi tempi di crescita in coltura. Per questo motivo negli ultimi anni le procedure per l'identificazione dei micobatteri hanno subito dei radicali cambiamenti. Alle convenzionali tecniche laboratoristiche, quali l' identificazione biochimica, sono

\section{Corresponding author: Raffaella Rossi}

Dipartimento di Malattie Infettive e Tropicali, "Sapienza"

Università di Roma, Policlinico Umberto I, 0016I Roma.

Tel.: 06-4997088I - Fax:06-49972625 - E-mail: raffaella.rossi@hotmail.it 
state affiancate nuove tecniche molecolari, sfruttando l'enorme progresso nel campo della biologia molecolare (1).

Sono disponibili in commercio vari kit per la tipizzazione dei MNT, tra cui il sistema AccuProbe (GenProbe, San Diego, CA) e il kit GenoType Mycobacterium (GenoType Mycobacterium; Hain, Nehren, Germany) che comprende due differenti pannelli: il GenoType Mycobacterium CM, che identifica le specie di MNT comuni e il GenoType Mycobacterium AS che identifica invece le specie di isolamento meno frequente $(4,9$, 10) (figura I). I kit sono validati su campioni estratti da colture in terreno liquido o solido.

Nell'ambito dell'infezione tubercolare, i primi studi sono stati eseguiti utilizzando il kit GenoType MTBDR (2) per la valutazione della resistenza a rifampicina ed isoniazide, tanto che oggi e oggi è stato validato un nuovo kit per queste resistenze direttamente su campioni respiratori con esame microscopico positivo per bacilli alcol-acido resistenti (GenoType Mycobacterium MTBDRplus) (8).

Utilizzando questo modello sperimentale, l'obiettivo del nostro studio è stato quello di valutare l'affidabilità dei kit GenoType Mycobacterium $\mathrm{CM} / \mathrm{AS}$ per la tipizzazione dei micobatteri atipici direttamente su campioni respiratori, al fine di ridurre drasticamente i tempi dell'iter diagnostico.

\section{MATERIALI E METODI}

Per lo studio sono stati analizzati quattro pazienti seguiti presso dal Dipartimento di Malattie Infettive e Tropicali, “Sapienza” Università di Roma. In tre di questi pazienti si riscontrava un forte sospetto clinico d'infezione da micobatteri atipici, mentre il quarto si stava sottoponendo alla terapia antitubercolare specifica senza però trarne alcun beneficio. Tutti i pazienti risultavano negativi per il test dell'HIV e tutti presentavano all'esame batterioscopico su espettorato bacilli alcool acido resistenti. Erano presenti come comorbidità bronco pneumopatia cronico-ostruttiva (BPCO) e/o immunodeficienza comune variabile.

È stato utilizzato il kit GenoType CM per la tipizzazione dei micobatteri atipici in campioni respiratori processati direttamente.

Il metodo molecolare GenoType si basa sull'ibridazione inversa (DNA strip) di un prodotto di PCR multiplex, su una strip di nitrocellulosa sul quale sono immobilizzate sonde specifiche per le diverse specie di micobatteri.

Il campione respiratorio è stato, quindi, decontaminato e centrifugato. Successivamente è stato risospeso in $0.1 \mathrm{ml}$ di acqua distillata sterile procedendo, poi, all'estrazione del DNA batterico. Dopo un'incubazione di 20 minuti in bagnomaria a $95^{\circ} \mathrm{C}$, il campione è stato sonicato per $15 \mathrm{~min}$ e quindi centrifugato, in modo da ottenere il DNA nel sovranatante. Si è quindi proceduto ad allestire una PCR come da protocollo del kit, aumentando da 20 a 30 il numero di cicli. La regione target che viene amplificata in PCR è la regione del gene che codifica per la subunità $23 \mathrm{~S}$ del ribosoma. Al termine dell'amplificazione, sul prodotto di PCR è stato eseguito il test GenoType CM come da protocollo del kit (www.hain-lifescience.com).

\section{RISULTATI}

I risultati del nostro studio mostrano come in tutti e quattro i pazienti arruolati siano presenti micobatteri atipici. In particolare sono stati tipizzati i seguenti micobatteri: $M$. peregrinum in due pazienti, $M$. gordone nel terzo ed infine $M$. intracellulare nel paziente in terapia antitubercolare.

Tutte le strip hanno 3 bande di controllo: la banda CC (controllo del coniugato) che ci conferma l'efficacia del legame del coniugato con il substrato; la banda UC (controllo universale) che rileva la presenza di tutti i micobatteri e del gruppo di batteri Gram positivi con un elevato contenuto di guanina e citosina nel genoma; la banda GC (controllo del genere) che documenta la presenza di un membro appartenente al genere Mycobacterium. I risultati del nostro studio sono stati in seguito confermati dalla crescita in coltura (tutti entro 10 giorni) di questi microrganismi. Utilizzando, quindi, lo stesso kit GenoType CM sono state eseguite le tipizzazioni dei micobatteri cresciuti in coltura. I risultati sono totalmente sovrapponibili, con una concordanza del 100\%. Nella figura II, infatti, è evidenziato un esempio rappresentativo in cui si può osservare nella prima strip la tipizzazione del $M$. peregrinum da campione respiratorio, mentre nella seconda strip la stessa tipizzazione da campione colturale. La differenza d'intensità delle bande nei due campioni è da attribuire alla quantità di micobatteri presenti nel campione di partenza: infatti, il campione di espettorato mostra bande meno intense rispetto ad un campione allestito con una colonia cresciuta in coltura. La significatività clinica dei micobatteri isolati (tabella 1) è stata valutata in base a quanto riportato nei criteri dell'ATS (5).

\section{DISCUSSIONE}

La diagnosi d'infezione da micobatteri a tutt'oggi risulta complessa ed eccessivamente lunga a causa del tempo necessario per lo sviluppo delle colonie in coltura. Gli enormi progressi nel campo genetico e molecolare, però, stanno dando una nuova impronta all'approccio diagnostico, basata non solo sulla sensibilità e specificità dei test ma anche sulla rapidità d'esecuzione, in modo da rag- 


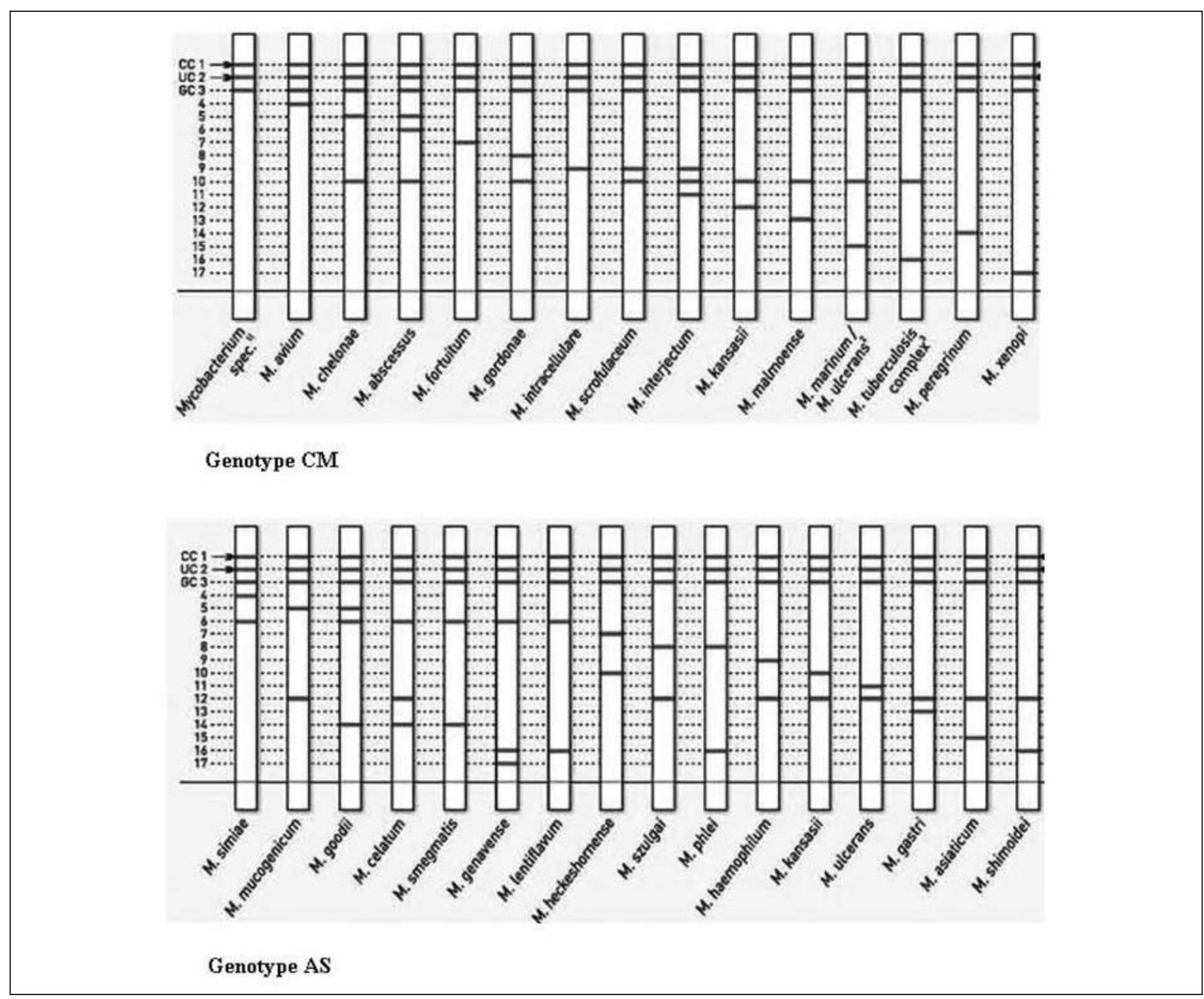

Figura I. Specie di micobatteri atipici che vengono evidenziate dai due kit in commercio. Il Genotype CM per le specie di micobatteri comuni e il Genotype AS per le specie addizionali

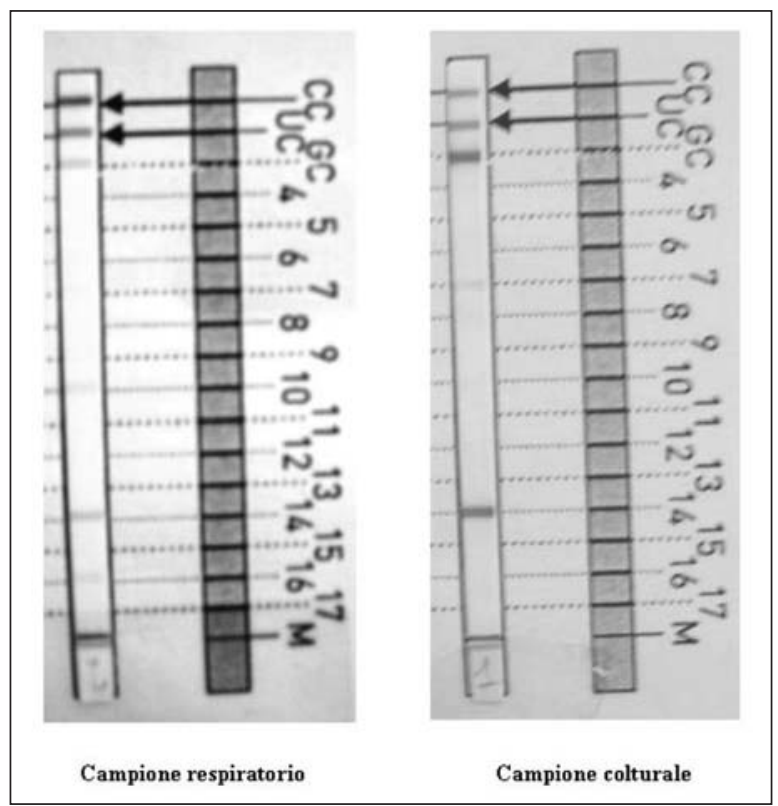

Figura II. Esempio rappresentativo di un confronto tra la tipizzazione del M. peregrinum eseguita da campione respiratorio e da campione estratto da una colonia cresciuta in coltura giungere una diagnosi eziologica in breve tempo e abbreviare notevolmente il lungo iter diagnostico delle infezioni da micobatteri.

Nell'ambito delle patologie da micobatteri non tubercolari, il nostro gruppo di ricerca ha pensato di utilizzare il protocollo per le resistenze ai farmaci antitubercolari $(2,8)$ sui kit GenoType $\mathrm{CM} / \mathrm{AS}$ per la tipizzazione dei micobatteri atipici $(4,9,10)$. Spesso, infatti, il clinico si ritrova a dover somministrare terapie antibiotiche dirette sia verso il $M$. tuberculosis che verso micobatteri atipici nell'attesa della tipizzazione successiva alla crescita colturale dei microrganismi.

I nostri dati mostrano chiaramente come arrivare ad una rapida diagnosi d'infezione da MNT utilizzando i kit GenoType CM/AS direttamente su campioni di provenienza respiratoria. I nostri risultati sono stati confermati in seguito dalla tipizzazione degli isolati ottenuti in coltura.

A nostro avviso risulta, quindi, molto importante continuare su questa linea di ricerca al fine di poter validare anche questi kit su campioni clinici. 
Tabella I. Caratteristiche generali della popolazione in studio

\begin{tabular}{|c|c|c|c|c|}
\hline & Paziente I & Paziente 2 & Paziente 3 & Paziente 4 \\
\hline Micobatterio isolato & M. peregrinum & M. peregrinum & M. gordone & M. intracellulare \\
\hline Sesso & $\mathrm{F}$ & $M$ & $\mathrm{~F}$ & $M$ \\
\hline Età (anni) & 38 & 70 & 56 & 78 \\
\hline Test HIV & - & - & - & - \\
\hline $\begin{array}{c}\text { Condizione } \\
\text { predisponente }\end{array}$ & $\begin{array}{l}\text { Immunodeficenza } \\
\text { comune variabile, } \\
\text { bronchiechesie }\end{array}$ & Diabete tipo II & $\begin{array}{c}\text { BPCO, } \\
\text { Mielodisplasia }\end{array}$ & BPCO \\
\hline Sintomatologia & Febbre, tosse & Tosse & Tosse, febbricola & Febbre, tosse \\
\hline RXoTAC & $\begin{array}{l}\text { TAC: Interstiziopatia } \\
\text { apicale bilaterale con } \\
\text { "albero in fiore", } \\
\text { multiple } \\
\text { bronchiechesie }\end{array}$ & $\begin{array}{c}\text { RX: } \\
\text { Interessamento interstiziale } \\
\text { medio-apicale del polmone } \\
\mathrm{dx} \text {, con formazione cavitaria } \\
\text { di } 10 \mathrm{~mm}\end{array}$ & $\begin{array}{c}\text { TAC: } \\
\text { Noduli polmonari } \\
\text { multipli, } \\
\text { bronchieclasie } \\
\text { bilaterali }\end{array}$ & $\begin{array}{c}\text { TAC: } \\
\text { Addensamento con } \\
\text { broncogramma aereo e } \\
\text { aree di cavitazione di } 10 \\
\text { mm nel lobo sup. sx. }\end{array}$ \\
\hline Batterioscopico & + & + & + & + \\
\hline Numero colture & 2 & 2 & 3 & 2 \\
\hline Campione & Espettorato & Espettorato & Espettorato & Espettorato \\
\hline Terapia & $\begin{array}{l}\text { Rifabutina, } \\
\text { etambutolo, } \\
\text { claritromicina }\end{array}$ & $\begin{array}{l}\text { Minociclina, } \\
\text { ciprofloxacina }\end{array}$ & $\begin{array}{l}\text { Rifabutina, } \\
\text { etambutolo, } \\
\text { ciprofloxacina }\end{array}$ & $\begin{array}{c}\text { Rifabutina, } \\
\text { etambutolo, azitromicina }\end{array}$ \\
\hline Durata terapia & 9 mesi & 9 mesi & 6 mesi & 6 mesi \\
\hline Significatività clinica & si & si & dubbia & si \\
\hline
\end{tabular}

Il progresso della genetica e della biologia molecolare potrebbe essere l'inizio di nuovi iter da utilizzare per la diagnostica delle infezioni da micobatteri che saranno senza dubbio di grande ausilio al clinico.

\section{BIBLIOGRAFIA}

1. Boden D, Weizenegger $\mathrm{M}$, Benz $\mathrm{K}$, et al. Reverse hybridization assay for rapid identification of Mycobacteria from cultured samples. Clin Lab 1998; 44: 687-92.

2. Cavusoglu C, Turhan A, Akinci P, Soyler I. Evaluation of the Genotype MTBDR assay for rapid detection of rifampin and isoniazid resistance in Mycobacterium tuberculosis isolates. $J$ Clin Microbiol 2006; 44: 2338-42.

3. Falkinham JO. Epidemiology of infection by nontuberculous mycobacteria. Clin Microbiol Rev 1996; 9: 177-215.

4. Gitti Z, Neonakis I, Fanti G, Kontos F, Maraki S, Tselentis Y. Use of the GenoType Mycobacterium CM and AS assays to analyze 76 nontuberculous mycobacterial isolates from Greece. J Clin Microbiol
2006; 44: 2244-6.

5. An Official ATS/IDSA Statement: Diagnosis, Treatment, and Prevention of Nontuberculous Mycobacterial Diseases. Griffith DE, Aksamit T, Brown-Elliott BA, et al. Am J Respir Crit Care Med. 2007; 175: 367-416.

6. Horsburgh CR. Epidemiology of mycobacterial diseases in AIDS. Res Microbiol 1992; 143: 372-7.

7. Horsburgh CR, Metchock B, Gordon SM, Havlik JA, McGowan JE, Thompson SE. Predictors of survival in patients with AIDS and disseminated Mycobacterium avium complex disease. J Infect Dis 1994; 170: 573-7.

8. Miotto P, Piana F, Migliori GB, Cirillo DM. Genotype MTBDRplus: a further step toward rapid identification of drug-resistant Mycobacterium tuberculosis. $J$ Clin Microbiol 2008; 46: 393-4.

9. Richter E, Rüsch-Gerdes S, Hillemann D. Evaluation of the GenoType Mycobacterium Assay for identification of mycobacterial species from cultures. J Clin Microbiol 2006; 44: 1769-75.

10. Russo C, Tortoli E, Menichella D. Evaluation of the new GenoType Mycobacterium assay for identification of mycobacterial species. J Clin Microbiol 2006; 44: 334-9.

11. Wolinsky E. Mycobacterial diseases other than tuberculosis. Clin Infect Dis 1992; 15: 1-10. 